\title{
Ambivalencia identitaria en la literatura chilena canónica*
}

\author{
Identity ambivalence in canonical chilean literature
}

\begin{abstract}
Iván Carrasco M.
Universidad Austral de Chile, Instituto de Lingüística y Literatura, Apartado 567, Valdivia, Chile, e-mail icarrasc@uach.cl
\end{abstract}

Considerando que la literatura ha sido valorada como un espacio muy particular e influyente de construcción identitaria por parte de escritores, críticos e investigadores, este trabajo estudia tres casos de identidad plural y ambivalente en un mismo personaje de autores y textos canónicos de la literatura chilena, Alberto Blest Gana, Pedro Prado y Vicente Huidobro. Los personajes son Martín Rivas, estudiado en cuanto metáfora sociopolítica, Alsino, interpretado como entidad compleja, simbólica y contradictoria, y Altazor, visto como representante de un tipo de líder artista y sacerdotal desacralizador.

Palabras clave: identidad, ambivalencia, líder, desacralización.

Considering that literature has been valued as a particular and influential space of identity construction by many writers, critics and researchers, this paper shows three cases of plural identity ambivalence in the same character by authors and canonical texts: Alberto Blest Gana, Pedro Prado and Vicente Huidobro. The characters are Martin Rivas who is studied in relation to a socio-political metaphor, Alsino, interpreted as a complex identity both symbolic and contradictory, and Altazor, representative of an artistic, as well as a priestly and paganizing leader.

Key words: identity, ambivalence, leader, paganizing.

\section{Fundamentos}

De modo expreso o implícito, la literatura ha sido considerada un espacio privilegiado de construcción identitaria. Umberto Eco ha afirmado que "La literatura, al contribuir a formar la lengua, crea identidades y comunidad" (2002: 11), Ricoeur, que las narraciones literarias son "modos mediante los cuales los individuos y los colectivos construyen sus identidades" (1985: 170), Sullá, que "el elenco de obras y autores sirve de espejo cultural e ideológico de la identidad nacional” (1998: 11), etc.

\footnotetext{
* Este trabajo forma parte del proyecto Fondecyt 1040321, realizado con la colaboración de mis colegas del Instituto de Lingüística y Literatura Drs. Oscar Galindo, Claudia Rodríguez y Ana Traverso, y fue leído en un congreso en la Universidad Católica de Valparaíso organizado por estudiantes y profesores de postgrado.
} 
En Latinoamérica la identidad ha sido investigada por su condición de continente colonizado, la persistencia de sus ancestros indígenas y los intensos procesos de mestizaje e interculturalidad desarrollados en distintos lugares y situaciones. Dorra ha manifestado que "En este panorama de cambios y permanencia que es la literatura vemos reaparecer, una y otra vez, el tema de la identidad cultural que se despliega como una tópica" /... " "toda gran obra surgida entre nosotros tiene que ver con nuestra identidad en la medida en que se incorpora profundamente a la cultura y termina por volverse un elemento esencial de su evolución. Toda gran obra habla siempre de nuestra identidad cultural, pues ayuda a constituirla" (2000: 132 y 137); R. Rojas, que "desde principios del siglo XIX aparecen discursos que intentan formalizar un canon de la cultura latinoamericana. En ellos se busca la definición de un sujeto histórico que traza su límite horizontal frente a los Estados Unidos y su límite vertical frente a Europa. La identidad latinoamericana recibe, desde entonces, una serie de formulaciones" (2000: 20); etc.

En general, la pregunta por la identidad se ha planteado en singular, como si sólo existiera la posibilidad de tener una identidad única, estable y nacional, lo que obviamente supone negar, invisibilizar o distorsionar otras existentes. Pero tanto el estudio empírico como algunas teorías identitarias actuales en nuestro país (Larraín 2000, Salazar 1999, Montecino 2003, p. ej.) señalan la existencia de variadas identidades en distintos niveles de la sociedad global. Desde esta perspectiva podemos considerar a muchas sociedades modernas como una serie de países socio y/o etnoculturales que coexisten en una especie de super o macropaís geográfico-administrativo, y por ello hay diferentes tipos de chilenos, es decir, de identidades (I. Carrasco 2003a: 391-96). Las identidades socioculturales o parciales no son alternativas, sino simultáneas, superpuestas, condicionadas y condicionantes en relación con las nacionales o supranacionales (Gissi 2002: 223).

En Chile la valoración de lo extranjero y la minimización de lo indígena y lo popular han configurado el tópico de una identidad europea, homogénea y moderna, aunque desde puntos de vista más plurales y menos etnocentristas existen posturas distintas; p. ej. en la Cartografía Cultural de Chile (1999) y en Revisitando Chile (2003), se reconoce la existencia de comunidades y grupos diferenciados que dan origen a identidades socioculturales definidas. Por lo demás, de hecho siempre hemos tenido variadas identidades en coexistencia, en el comienzo tres muy nítidas de origen etnocultural: la indígena, en sus variedades mapuche, aymara, chono, selknam...; la europea en sus versiones española, italiana, alemana...; y la criolla. Algunas han desaparecido, como la de los realistas, los misioneros de indios; otras están en disminución y transformación, como la de rotos y huasos, y algunas afirmándose con fuerza, como las de género, en particular la feminista. Junto a ellas existen otras proscritas en los discursos dominantes: las etáreas, las interculturales, las religiosas de avanzada, los grupos marginales, para mencionar algunas.

Nuestra conjetura es que la literatura chilena canónica presenta una marcada y compleja ambivalencia identitaria que se desarrolla en dos planos, uno que coincide con las existentes en la vida histórica y social del país, y otro que no guarda relaciones de reproducción o analogía con ellas. El primer tipo se funda en la capacidad representativa o mimética de la literatura, mientras que el segundo se basa en su condición perlocutiva, que le permite proponer identidades distintas a las existentes al mismo tiempo que oponerse a los cánones e ideologías predominantes. En los dos planos no 
aparece una sola identidad, sino distintas identidades, variables y a veces opuestas, establecidas por procesos superpuestos de canonización.

Esto es observable en los discursos metaliterarios o autorreflexivos de la literatura chilena y en los cánones que rigen la producción, recepción y desarrollo de las literaturas en relación implícita o explícita con las identidades existentes o soñadas en esa cultura. En los textos literarios chilenos se puede observar una especie de oscilación entre modelos extranjeros, nacionales e interculturales de identidad (I. Carrasco 2001, Rodríguez 2007). La polivalencia entre la fascinación por lo extranjero, europeo o exótico, que se siente prestigioso, de mayor calidad y universal, y la depreciación de los modelos de lo propio, subvalorados aunque reconocidos como auténticos, se expresa como simultaneidad y superposición más que como dicotomía. Esta actitud connota una identidad nacional inestable, ambivalente, intercultural pues debilita o reconstruye la oposición entre nacionalismo y extranjerismo, presente sobre todo en los momentos conflictivos de nuestra historia. Las posturas nacionalista o extranjerizante responden a un anhelo de ocultar el carácter mestizo y multicultural de la nacionalidad chilena y también nuestro negado etnocentrismo. El reconocimiento de lo mapuche como parte del discurso público nacional (H. Carrasco 1996 y 2002) y de una interculturalidad efectiva, apunta hacia la esperanza de la solidaridad etnocultural a partir del respeto y la aceptación del otro indígena.

En este trabajo examinaremos las identidades representadas en tres textos canónicos caracterizados por protagonistas singulares que encarnan más de una identidad y constituyen homónimos de los protagonistas y las obras en que aparecen: Martín Rivas, Alsino y Altazor. Los antecedentes de este análisis y un desarrollo mayor se encuentran en los resultados de una investigación y en un libro en preparación sobre los problemas de las relaciones entre identidades y canonizaciones en la literatura chilena.

\section{MARTÍN RIVAS: ENTRE LA REBELDÍA Y LA INTEGRACIÓN}

"Tal como la literatura chilena del siglo XVII es el Padre Ovalle y Pineda Bascuñán, dentro de doscientos años nuestro siglo XIX se reducirá, probablemente, a Pérez Rosales y Blest Gana: el hombre del libro único, personalísimo, y el autor de una vasta y múltiple construcción novelesca bastarán para representarnos ante la posteridad" /.../ Blest Gana es en nuestra historia un Padre de la Patria" (Alone 1954: 179 y 185).

En los estudios e historias de la literatura chilena siempre ha aparecido José Victorino Lastarria como su punto de partida, aunque no se puede negar que sus textos fundamentales son metatextos programáticos (Promis 1995: 20-27) y su obra propiamente artística no ha alcanzado valoración ni proyección equivalentes a aquellos.

En cambio, Alberto Blest Gana es el primer autor canónico de la literatura chilena; su obra ha sido leída, estudiada y comentada por una gran variedad de lectores que la han mantenido en vigencia hasta ahora y puede ser considerada un muestrario de identidades de su época. De hecho, en ella aparecen las tres identidades históricas básicas de Chile: la indígena mapuche, realzada en Mariluán; la europea realista o monárquica, en Durante la Reconquista, donde también destacan la identidad criolla en sus manifestaciones patriota (Manuel Rodríguez, Abel Malsira y su bella hermana) 
y popular, encarnada en el roto y el huaso, o el siútico (rastaquouère) en su versión patricia disipada o popular.

La novela canónica de Blest Gana es Martín Rivas, uno de los libros más editados, leídos y comentados de la narrativa chilena, "la obra que lo conduciría definitivamente a la fama" y su protagonista homónimo "Uno de los pocos personajes de la narrativa nacional que se mantiene en el recuerdo de los lectores" (Fernández 1996: 257).

Lo que se ha canonizado en el personaje y en la novela es la identidad de clase media: ha sido considerado héroe de la clase media (Melfi 1938: 9), el triunfo de la clase media (Alone 1940: 163), símbolo de la clase media (Guerrero 1967: 11), etc., postura que establece una relación mimética entre esta novela y la sociedad chilena.

Pero también hay en el personaje Rivas una extraña ambivalencia: por una parte logra su anhelo de mejorar su condición económica y social, lo que consigue mediante sus valores morales, los servicios a sus protectores, la conquista de Leonor y hacerse cargo de los negocios de la familia Encina, con lo cual se convierte en aliado de la clase alta. Martín ha luchado contra esta mediante la defensa (parcial) de su condición provinciana y el apoyo de una ideología diferente al seguir los ideales revolucionarios de su amigo Rafael San Luis y participar en el motín de Urriola.

De acuerdo a esto, Rivas representa a la clase media, sí, pero en el momento de su instauración ideológica y no de su consolidación económica, como simpatizante de un conglomerado específico en gestación dentro de ella, el radicalismo; en este sentido, la novela es vehículo ideológico que difunde la mentalidad que surge. (Concha 1972). A través de la dedicatoria a Manuel A. Matta y del realce dado al motín de Urriola, hecho histórico que sirve de trasfondo a las acciones claves de la novela, el autor ha mostrado su ambigüedad: Martín Rivas representa una identidad vigente, la burguesía liberal, pero al mismo tiempo anuncia una modificación sustantiva de ella que aún no se ha realizado.

Como escribió Melfi (1938: 32) y subrayó Concha, Martín Rivas es cifra y sello de una clase en formación. Pero se establece ambiguamente en relación a su proyecto identitario, pues al mismo tiempo que señala una crisis en un sector de ella, oscila entre la fidelidad a su condición sociocultural y el deseo arribista de lo ajeno mediante el amor. Y, en ese sentido, aparece más de una identidad en un personaje y torna ambigua la relación mimética texto-contexto.

\section{Alsino: ENTRE LA TIERRA Y El CIELO}

Esta obra de Pedro Prado es considerada una de las más hermosas y originales de la narrativa chilena. Torres-Ríoseco ha dicho que es una de las más bellas producidas en nuestro continente, lo que ha sido ratificado por Silva Castro, "La novela Alsino es, según la opinión de varios autorizados críticos, la más ambiciosa creación intelectual que ofrece la literatura hispanoamericana en el período moderno" (en Alegría 1959: 138) y por Alegría, que la considera una de las más excelsas composiciones alegóricas latinoamericanas (id: 139). Según Alone es su obra capital o considerada capital, una especie de novela poética o poema en prosa y su propósito es crear un símbolo, la expresión idealista infiltrada en los discursos prácticos, positivos, cercanos al instinto puramente zoológico (1962: 302). Por su parte, Maximino Fernández afirma que "es la obra más famosa de Prado y tal vez la única que funda un mito" (1996: 415). 
Para Melfi se trata de un símbolo en que se mezclan la realidad chilena del campo y el idealismo: "Alsino es la novela poemática de la ascensión espiritual; la elevación del hombre inculto que se transfigura por el contacto de las bellezas adyacentes, se embriaga de infinitud y de pureza, pero al que la vida externa, dura y contradictoria, combate sin piedad" (1938: 16); Silva Castro, se refiere a la vieja inquietud humana por volar que la atribuye al poeta: reconoce en el texto "un profundo símbolo/.../ la preferencia de Alsino por cantar, cuando suspendido de sus alas flota en el aire. Al decir esto, Prado nos está sugiriendo como el poeta necesita encontrarse desasido de la pesantez que le ata a la tierra para atreverse a dar expresión al cantar poético" (1961: 260). Según Arriagada y Goldsack, Alsino es una alegoría social: "Sorprende, en efecto, que haya elegido como héroe de su creación, no a un robusto mocetón o a un joven intelectual, sino a un muchachito analfabeto, sobre cuyo destino se cierne el mismo que hoy por hoy amenaza las reservas vitales de la raza: el alcoholismo. El hecho de que haya preferido a los campesinos y no a los trabajadores industriales de las ciudades, debe explicarse por la fisonomía del anarquismo místico tan de moda en los días de su formación /.../ la juventud buscaba el retorno a la sencillez de los primeros cristianos en conformidad al pensamiento tolstoyano. En "Alsino", más que un ansia de liberación colectiva, campea el deseo de libertar al individuo de todas las trabas del Estado y de la sociedad, para convertirlo en una victoriosa autarquía/.../ Alsino es la glorificación de un vagabundo ideal" (1952: 71-2).

Los diversos enunciados canonizantes de los estudiosos de Prado que hemos escogido destacan el carácter simbólico del personaje, un personaje de naturaleza compleja, dual, como intuyó Alone (1962: 170), aunque algunas interpretaciones carecen de base. Desde una perspectiva identitaria sociocultural, el personaje Alsino es un ser contradictorio que incluye rasgos de distintos modos de ser. Al comienzo, es un niño que vive junto a sus padres alcohólicos en un ambiente miserable que anula sus posibilidades de desarrollo. Pero, este primer Alsino no representa la identidad campesina, sino la de un estrato social que vive entre el campo y la playa en contacto con pequeños poblados aislados y manteniéndose con una economía de subsistencia, viviendo al día. Es la identidad marginal, que se explica en esta obra desde una óptica naturalista.

El accidente que provoca el milagro de dotarlo de alas y lo transforma en un ser extraordinario, oscila entre el milagro, la hierofanía y la metáfora: Alsino vuela, canta, se comunica con los animales, cura enfermos y entrega mensajes de sabiduría y amor; es una mezcla de personaje mítico mientras vuela, canta y habla con aves, animales y otros seres naturales, se parece a Jesús cuando es seguido por muchedumbres que esperan salud y salvación, Alsino también soporta la injusticia y el dolor; no deja de ser humano, pero no es Dios, muere y no resucita, representa una identidad ambivalente y ambigua. Su extraña condición crea la admiración pero también el miedo y el rechazo de los seres comunes que temen su superioridad. A diferencia del niño, ya no sueña pues su sueño es realidad y le permite vivir momentos extraordinarios, aunque no permanentes ni sagrados pues no lo defienden de la maldad ni de la muerte.

El personaje Alsino encarna identidades contradictorias, la del niño y la del ser extraordinario. Aunque se puede confundir con el modelo de anarquista cristiano de origen bíblico y literario de comienzos de siglo, no coincide en un aspecto central: aquel elegía voluntariamente su vida de compromiso con la justicia y la libertad, no así Alsino que está determinado por la herencia y el medio a ser lo que era.. 
$\mathrm{Al}$ incluir en la novela esta complejidad ambivalente, Prado rompe la verosimilitud identitaria propia de la modernidad, pues las identidades que encarna Alsino carecen de coherencia entre ellas y Alsino carece de semejanza con los seres humanos normales; esto problematiza la simetría entre persona e identidad única, al mismo tiempo que la analogía entre arte y realidad.

\section{AltaZor: LÍDER POETA SACERDOTAL/SECUlARIZAdo}

“La poesía contemporánea tiene sus primeras manifestaciones hacia 1915 o 1916 con una dirección mantenida y consciente en la obra de Vicente Huidobro publicada en América, en Francia o en España y difundida con eficacia a lo largo de los años veinte en todo el mundo hispánico" (Goic 1988: 74); "Contradictorio, megalómano, egocéntrico, inteligente, audaz, apasionado y figura controvertida, Vicente Huidobro es uno de los pilares de la literatura en lengua española en el siglo XX" (Pérez López 1998: 13). Para Yurkievich, es uno de los fundadores de la nueva poesía latinoamericana, su talento se equipara con el de Vallejo y Neruda, con quienes integra el tríptico mayor de la poesía contemporánea. (1971: 56). Más admirativo que todos, Paz ha afirmado que Huidobro "contempla de tan alto que todo se hace aire. Está en todas partes y en ninguna: es el oxígeno invisible de nuestra poesía” (1993: 96).

Enunciados canonizadores como estos se encuentran en los mejores especialistas de literatura contemporánea, que alaban al poeta, al promotor del vanguardismo, al esteta. Poetas y críticos coinciden en que el poema extenso Altazor es su mejor realización. Baciu, p. ej., afirma que "no sólo es su creación máxima, sino también una de las más importantes obras de la poesía latinoamericana de todos los tiempos" (1974: 68).

A menudo la crítica tiende a establecer una relación analógica o metafórica entre autor y texto, realidad y ficción, historia y arte, por lo cual lo que se afirma de Altazor es lo que se piensa de Huidobro, o a la inversa; Jimeno distingue la diferencia y destaca la conexión, pero también enfatiza al autor: "Altazor y Temblor de cielo son los dos vértices de tensión que conduce la corriente de la energía vital y estética donde se entrecruzan vida y arte. Como Pound, Joyce, Rilke, Hesse, Pirandello y sobre todo Pessoa, Huidobro es una personalidad con la dispar unidad de varias personas en un solo personaje" (en Goic 2003: 1479). Su interesante observación podría ser válida como imagen de la figura pública de Huidobro, pero parece tener más pertinencia para representar la identidad del personaje Altazor, quien, según proposición anterior de Goic (1992: 43) presenta una compleja suma de dimensiones contradictorias. Concha también había considerado a Altazor un personaje híbrido (ente humano, semipájaro, quizás un ángel por su don de vuelo), de personalidad plural, en quien se conjugan la condición natural de vate y de juglar (1965).

Altazor es una entidad cósmica, multiforme, sobrehumana, al decir de Goic, un ente despersonalizado que excede las posibilidades y capacidades de lo humano, cuyo comportamiento muestra una dimensión humana constantemente sacada de su condición usual por una torsión magnificadora, desmesurada o cósmica (1992); es una metáfora continua según Yurkievich (1997: 140).

Desde nuestra perspectiva, Altazor es un ser plural, conformado por identidades distintas, contradictorio con cualquier sujeto social y por ello imposible en la vida 
histórica, debido a la concepción creacionista que lo ha conformado. Por lo tanto, no puede definirse como mera analogía o reflejo de Huidobro en sentido estricto. La confusión entre sujeto autor y personaje depende de la "oscilación enunciativa" (Carrasco 2003b, en Goic 2003: 1551) que caracteriza algunos textos fundamentales como "El paso del retorno" y Altazor, en que la palabra del vate cambia sin cesar de destinatario y de punto de vista, provocando una sensación de movimiento, vuelo, fuerza y confusión propio del fervor y la euforia del viaje maravilloso.

La identidad principal del relato lírico es la del líder, derivada de su actitud cercana a Zaratustra, cósmica, sobrehumana, perceptible en el apóstrofe permanente a vastas multitudes o entidades grandiosas, el afán desacralizador de seres poderosos, el ritual iniciático, la postura arrogante, segura de sí mismo y de ser el conductor de la humanidad, el ansia de una mujer también sobrehumana (Canto II), su voluntad permanente de autodefinición: "Soy la voz del hombre que resuena en los cielos/.../ Soy todo el hombre" (vv. 375 y 378). Esta identidad dominante coexiste con otra, con la sacral que busca y reniega de la divinidad, que se juega en la relación con/ contra ella: "Señor Dios si tú existes es a mí a quien lo debes" (v. 450), en su fusión jánica con el modo de ser del hombre secularizado de la modernidad: "Abrí los ojos en el siglo en que moría el cristianismo” /.../, “ ¿Y mañana qué pondremos en el sitio vacío? (vv. 100 y 104). Pero en su subjetividad privilegiada también vive un poeta artista creador revolucionario esteticista transgresor: poseedor de lo indecible: "Tanta exaltación para arrastrar los cielos a la lengua/ El infinito se instala en el nido del pecho/ Todo se vuelve presagio/ ángel entonces/ el cerebro se torna sistro revelador/ y la hora huye despavorida por los ojos" (vv. 650-55).

\section{OBRAS CITADAS}

Alegría, Fernando. 1959. Breve historia de la novela hispanoamericana. México: Ediciones de Andrea.

Alone. 1940. Don Alberto Blest Gana. Santiago: Nascimento.

- 1954. Historia personal de la literatura chilena. Santiago: Zig-Zag; $2^{\mathrm{a}}$ ed. 1962. Los cuatro grandes de la literatura chilena. Santiago: Zig-Zag.

Arriagada, Julio y Goldsack, Hugo. 1952. Pedro Prado, un clásico de América. Santiago: Nascimento.

Baciu, Stefan. 1974. Antología de la poesía surrealista latinoamericana. México: Joaquín Mortiz.

Badal, Gonzalo. 1999. (ed.). Cartografía Cultural de Chile. Atlas. Santiago: MINEDUC, División de Cultura.

Carrasco, Hugo. 1996. "El discurso público mapuche". Lengua y Literatura Mapuche 7: 105117.

. 2002. "El discurso público mapuche: noción, tipos discursivos e hibridez". Estudios Filológicos 37: 185-97.

Carrasco, Iván. 2001. "Pluralidad y ambivalencia en la metatextualidad literaria chilena", Estudios Filológicos 36: 9-20.

. 2003a. “QQué significa ser chilenos en uno de los sures de Chile?”, en Montecino, Sonia. Revisitando Chile..., 391-96.

2003b. "Ultimos poemas: la voz que no decrece”, en Goic 2003..., 1547-1558. 
Concha, Jaime. 1965. "Altazor de Vicente Huidobro”. AUCh 133: 113-168.

—. 1972. "Martín Rivas o la Formación del Burgués". Revista Chilena de Literatura 5-.6: 9-36.

Dorra, Raúl. 2000. "Notas sobre el tema de la identidad iberoamericana". Hablar de literatura. México: FCE, 127-37.

Eco, Umberto. 2002. Sobre Literatura. Barcelona: RqueR Ediciones.

Fernández, Maximino. 1996. Historia de la Literatura Chilena. Santiago: Editorial Salesiana; $2^{\mathrm{a}}$ ed.; 2 Tomos.

Gissi, Jorge. 2002. Psicología e identidad latinoamericanas. Sociopsicoanálisis de cinco Premios Nobel de Literatura. Santiago: Ediciones Universidad Católica de Chile.

Goic, Cedomil. 1988 y 1991. Historia y crítica de la literatura hispanoamericana. Barcelona: Crítica, Tomos III y II.

—.1992. Los mitos degradados. Ensayos de comprensión de la literatura hispanoamericana. Ámsterdam-Atlanta: Rodopi.

— 2003 (Coord.). Vicente Huidobro. Obra Poética. Edición crítica. Madrid: ALLCA XX; Colección Archivos 45.

Guerrero, Leoncio. 1967. "Prólogo" a Martín Rivas. Buenos Aires: Orbe, 9-12.

Jimeno Grendi, Orlando. 2003. "Modernidad y fragmentación de la conciencia en Temblor de cielo", en Goic 2003..., 1464-479.

Larraín, Jorge. 2001. Identidad chilena. Santiago: LOM.

Melfi, Domingo. 1938. Estudios de Literatura Chilena. Primera serie. Santiago: Nascimento.

Montecino, Sonia (Comp.). 2003. Revisitando Chile. Identidades, mitos e historias. Santiago: Cuadernos Bicentenario.

Paz, Octavio. 1993. El arco y la lira. México; FCE; Novena reimpresión.

Pérez López, María Ángeles. 1998. Los signos infinitos. Un estudio de la obra narrativa de Vicente Huidobro. Lleida: Edicions de la Universitat de Lleida.

Promis, José. 1995. Testimonios y documentos de la literatura chilena. Santiago: Andrés Bello.

Ricoeur, Paul. 1985. Ideología y utopía. Barcelona: Gedisa.

Rodríguez, Claudia. 1997. "Del Parnaso a la Cordillera. Metatextos fundacionales (1842), Estudios Filológicos 42: 203-14.

Rojas, Rafael. 2000. Un banquete canónico. México: FCE.

Salazar, Gabriel y Pinto, Julio. 1999. Historia contemporánea de Chile II. Actores, identidad y movimiento. Santiago: LOM Ediciones.

Silva Castro, Raúl. 1958. 1961. Panorama literario de Chile, Santiago: Universitaria.

Sullá, Enric (comp.). 1998. El canon literario. Madrid: Arco/ Libros.

Yurkievich, Saúl. 1971. Fundadores de la nueva poesía latinoamericana. Vallejo Huidobro.

Borges Neruda Paz. Barcelona: Barral Editores.

—— 1997. Suma Crítica. México: FCE. 